\title{
Topological edge states in Rashba-Dresselhaus spin-orbit-coupled atoms in a Zeeman lattice
}

\author{
C. Li, ${ }^{1,2}$ F. Ye, ${ }^{1 *}$ X. Chen, ${ }^{1}$ Y. V. Kartashov, ${ }^{3,4}$ L. Torner ${ }^{3,5}$, V. V. Konotop ${ }^{6}$ \\ ${ }^{1}$ Key Laboratory for Laser Plasma (Ministry of Education), Collaborative Innovation Center of \\ IFSA, School of Physics and Astronomy, Shanghai Jiao Tong University, Shanghai 200240, China \\ ${ }^{2}$ School of Physics and Optoelectronic Engineering, Xidian University, Xi'an 710071, China \\ ${ }^{3}$ ICFO-Institut de Ciencies Fotoniques, The Barcelona Institute of Science and Technology, \\ 08860 Castelldefels (Barcelona), Spain \\ ${ }^{4}$ Institute of Spectroscopy, Russian Academy of Sciences, Troitsk, Moscow Region, 108840, Russia \\ ${ }^{5}$ Universitat Politecnica de Catalunya, 08034 Barcelona, Spain \\ ${ }^{6}$ Departamento de Física and Centro de Física Teórica e Computacional, \\ Faculdade de Ciências, Universidade de Lisboa, Campo Grande, Ed. C8, Lisboa 1749-016, Portugal \\ *Corresponding author: fangweiye@sjtu.edu.cn
}

The last decade has witnessed continuously growing interest in the investigation of spin degrees of freedom in various systems in solid-state physics, atomic physics, optics, and acoustics. The dynamics of spin (or quasi-spin) becomes especially intriguing when it interacts with other degrees of freedom. A well-known example of such interaction is the coupling between spinor and translational degrees of freedom, known as spin-orbit coupling. One of the most spectacular manifestations of the spin-orbit coupling is the appearance of in-gap topological edge states at the boundaries or interfaces between periodic structures. Such topological edge states are particularly robust because they are immune to weak disorder and to absence of backscattering by surface defects. Novel prospects for the exploration of the physics of topological edge states and insulators open in systems of neutral atoms placed in periodic potentials, where diverse gauge potentials can be artificially created. Here, we suggest a new platform, where topological edge states emerge due to the interplay between spin-orbit coupling and a Zeeman lattice, characterized by opposite signs for the spinor components. We illustrate strong effect of different components of spin-orbit coupling on the emergence of the topological states. We also obtain nonlinear edge states and study their instabilities in the presence of interatomic interaction in spin-orbit coupled Bose-Einstein condensates.

Discrete and continuous lattices exhibit degeneracies in the eigenmode spectrum when the corresponding Hamiltonian is characterized by suitable spatial symmetries and time-reversal invariance [1]. Graphene, as the paradigm of a honeycomb lattice [2], is one of the best-known examples of structures where energy bands touch at Dirac points. If the underlying symmetries are broken, a gap may open at the Dirac points thus leading to a transition to either a conventional or a topological insulator phase, depending on which symmetry is broken [1]. When such a lattice is located in contact with a material having distinct topological properties, topological states with energies falling into the gap and localized at the edge between two materials may appear. An outstanding perturbation that leads to the appearance of topological edge states is spin-orbit coupling (SOC), which in electronic systems gives rise to the quantum spin Hall effect [3, 4].

Interest in topological edge states is constantly growing [4, 5] and to date the concept of topological insulation has been extended to several areas of physics, where SOC can be emulated by coupling the translational and the internal spinor degrees of freedom, the latter often referred as pseudo-spin. Topological insulators have been realized in acoustic [6] and mechanical systems [7], as well as in optical and optoelectronic systems [8], including gyromagnetic photonic crystals [9-11], semiconductor quantum wells [12], arrays of coupled resonators [13, 14], metamaterial superlattices [15], helical waveguide arrays [16-18], systems with driving fields containing vortex lattices [19], and in polaritonic systems, where SOC originating in splitting of energy levels for different polarization states leads to topological effects [20-22]. Simulation of quantum spin Hall effect with atoms in optical lattices subject to the field gradient was proposed in [23].

A synthetic SOC can be simulated in atomic systems [2427], where proper superpositions of hyperfine atomic states, which are described by two- (or multi-) component wavefunctions, have spinor character, and therefore mimic spin. Such states are considered to bear a pseudo-spin. In atomic systems with the SOC induced by an homogeneous field, the spin Hall effect was observed experimentally [28].

In this paper we show that one can realize topological edge states in systems of cold pseudo-spin-1/2 SOC atoms and SOC Bose-Einstein condensates (SO-BEC) [24, 25] embedded in a Zeeman lattice. Our proposal employs periodically-varying Zeeman splitting induced by the external fields thus leading to the formation of a lattice. Such lattices having opposite signs for two spinor components may obey a desirable symmetry [29] and are feasible experimentally [30]. The peculiarity of Zeeman lattice is that when spinor components are decoupled, one of them is localized in deep potential wells which can be described by the tight-binding approximation, while the second component is in the "almostfree-electron" limit. SOC links spinor components, i.e. it couples two atomic states with practically opposite dynamical properties. Such setting affords the exploration of the effect of different types of SOC, which are tunable in atomic systems [25], where nearly arbitrary gauge potentials can be 
created $[26,27]$. In particular, one can explore the interplay of the Rashba [31] and Dresselhaus [32] mechanisms in the formation of topological edge states. When considering SOBEC, the nonlinearity stemming from two-body interactions, becomes relevant. The effect of two-body interactions can be twofold. On the one hand, it can affect the mode structure, even facilitating emergence of topological states, as it was recently reported in [33] for harmonically trapped spin-one spinor BEC in a honeycomb lattice. On the other hand, the nonlinearity couples all modes, including topological ones, that can lead to different kinds of dynamical instabilities of the states obtained in stationary analysis. Since the scattering length of the two-body interactions in a BEC can be both positive and negative, one can explore the behavior and stability of topological edge states in different nonlinear regimes.

\section{Results}

Model. We address a SOC atom described by the spinor $\boldsymbol{\psi}=\left(\psi_{1}, \psi_{2}\right)^{T}$ (T stands for the transpose), whose evolution is governed by the Schrödinger equation (we use units where $m=\hbar=1$ )

$$
i \frac{\partial \boldsymbol{\psi}}{\partial t}=\hat{H} \boldsymbol{\psi}, \quad \hat{H}=\hat{H}_{0}+\hat{H}_{\mathrm{SOC}}
$$

Here,

$$
\hat{H}_{0}=\left(\hat{k}_{x}^{2}+\hat{k}_{y}^{2}\right) / 2+\hat{U}(\mathbf{r}),
$$

with $\hat{k}_{x}=-i \partial_{x}$ and $\hat{k}_{y}=-i \partial_{y}$, describes the spinor in the external potential $\hat{U}(\mathbf{r})$. The SOC is described by the Hamiltonian

$$
\hat{H}_{\mathrm{SOC}}=\beta_{y} \sigma_{x} \hat{k}_{y}-\beta_{x} \sigma_{y} \hat{k}_{x}
$$

where the positive constants $\beta_{x, y}$, are determined by the interplay between Rashba SOC and Dresselhaus SOC, and $\sigma_{x, y, z}$ are the Pauli matrices. The case $\beta_{x}=\beta_{y}=\beta_{\mathrm{R}}$ corresponds to the pure Rashba SOC $\beta_{\mathrm{R}}\left(\sigma_{x} \hat{k}_{y}-\sigma_{y} \hat{k}_{x}\right)$ [31]. Similar gauge potentials were previously used in atomic systems [34, 35], where they are created by the external coupling of hyperfine states. This flexibility is a crucial difference between atomic systems and their solid-state counterparts, where the particular form of SOC depends on the symmetry of the crystalline lattice [36, 37]. Independently engineered Zeeman lattice [30], is created to have identical functional shapes, but opposite signs for two different spinor components. It is modeled by the potential $\hat{U}(\mathbf{r})=-\mathcal{R}(x, y) \sigma_{z}$, where

$$
\mathcal{R}(x, y)=\rho \sum_{m, n} e^{-\left[\left(x-x_{m}\right)^{2}+\left(y-y_{n}\right)^{2}\right] / d^{2}}
$$

describes a honeycomb structure with the amplitude $\rho$, characteristic width, $d$, of lattice sites at the nodes $\left(x_{m}, y_{n}\right)$ of the discrete honeycomb grid. The distance between neighboring sites is $a$. In these notations $-\mathcal{R}(x, y)$ and $+\mathcal{R}(x, y)$ are the potentials for the components $\psi_{1}$ and $\psi_{2}$, respectively. Thus, the spatial domains, where $\psi_{1}$ tends to have maxima are the regions with strongest expulsion for $\psi_{2}$. This leads to nontrivial competition between Zeeman lattice and SOC, since the latter tends to create nonzero density in $\psi_{2}$ component in the vicinity of density maxima of $\psi_{1}$ component, i.e. around maxima of potential for $\psi_{2}$.

We assume that the Zeeman lattice is infinite along the $y$-axis and is truncated along the $x$-axis. The truncation is such that the lattice has two different edges, where topological states can appear: zigzag (left) and bearded (right) ones. Such truncation allows us to compare directly the properties of the modes excited at the edges of different types. The top row of Fig. 1 shows representative lattice shape used in the simulations. The modes of such truncated lattice are Bloch waves of the form $\boldsymbol{\psi}(x, y)=e^{i k y-i \varepsilon(k) t} \phi(x, y)$, where $\varepsilon(k)$ is the energy, $k \in[0, K]$ is the Bloch momentum along the $y$-axis, $\phi$ is the periodic spinor function with a period $L=3^{1 / 2} a$ along the $y$-axis, and $K=2 \pi / L$ is the width of the Brillouin zone.

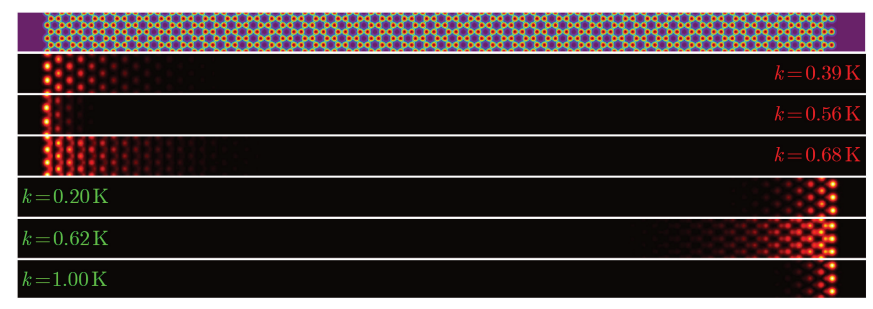

FIG. 1. Linear edge states. Upper panel: the Zeeman lattice with zigzag (left) and bearded (right) edges used in simulations (three unit cells are shown). Lower panels: examples of edge states for different Bloch momenta indicated in the panels. Only $\left|\psi_{1}\right|$ is shown. In all cases $\beta_{x}=\beta_{y}=1.5$.

Linear topological edge states. In the absence of SOC, i.e. when $\beta_{x}=\beta_{y}=0$, the spinor component $\psi_{j}(j=1,2)$ solves the stationary Schrödinger equation with the potential $(-1)^{j} \mathcal{R}(x, y)$. Since the potential $-\mathcal{R}(x, y)<0$ represents an array of narrow wells, the ground state of the $\psi_{1}$ component has negative energy. The potential $+\mathcal{R}(x, y)$ is positive and corresponding energy spectrum for $\psi_{2}$ is located at $\varepsilon>0$. This peculiarity of the Zeeman lattice has several important consequences. First, in the presence of weak SOC the opening of the lowest topological gap is expected at those (negative) energy levels, where degeneracies in the form of Dirac points are encountered in the spectrum of $\psi_{1}$ when it is decoupled from $\psi_{2}$. Second, in this lowest topological gap all states are characterized by dominating $\psi_{1}$ component. Finally, for a deep lattice, like the one used here within the framework of the full continuous model, the decoupled component $\psi_{1}$ is well described by the tight-binding approximation [38].

One of our central findings is that the type of SOC, or more precisely the relation between SOC strengths $\beta_{x}$ and $\beta_{y}$, is a decisive factor that determines whether topological modes can be created. Indeed, let us first consider the case when one of the SOC components is nonzero, but small enough to be considered as a perturbation of the Hamiltonian $\hat{H}_{0}$ of the infinite lattice. Let $\mathcal{T}$ be the time reversal operator that changes $\hat{\mathbf{k}} \rightarrow-\hat{\mathbf{k}}$ and performs complex conjugation of the wavefunction: $\mathcal{T} \psi(\mathbf{r})=\psi^{*}(\mathbf{r})$. The gap in the spectrum of the infinite lattice can open only in the vicinity of the Dirac points under the action of perturbations. However for a pair of Dirac points 
with coordinates $[0, \pm 2 K / 3]$ in the reciprocal lattice, the SOC component $\beta_{x} \sigma_{y} k_{x}$, which does not break time-reversal symmetry $\mathcal{T}$, becomes exactly zero, since in these points $k_{x}=0$. Thus, total gap cannot be opened by such a perturbation. On the other hand, perturbation introduced by other SOC component $\beta_{y} \sigma_{x} k_{y}$ that acquires nonzero value $\beta_{y} \sigma_{x}(2 K / 3)$ in the above mentioned Dirac points, does open the gap. We have verified these properties numerically not only for small values of SOC strengths, but also for $\beta_{x} \sim 1$ and $\beta_{y} \sim 1$ (keeping $\beta_{y}=0$ or $\beta_{x}=0$, respectively). Even though $\beta_{y} \sigma_{x} \hat{k}_{y}$ perturbation opens the gap around Dirac points of $\hat{H}_{0}$ and even though it is neither $\mathcal{T}$ - nor $\mathcal{P}$-symmetric (where $\mathcal{P}$ is the operator of spatial inversion), it cannot lead to appearance of topological states, when it acts alone. The reason behind this is that unperturbed Hamiltonian $\hat{H}_{0}$ obeys additional time reversal symmetry $\mathcal{T}^{\prime}=\sigma_{z} \mathcal{T}$ that is equivalent to introduction of unessential phase factor $\psi_{2} \rightarrow e^{i \pi} \psi_{2}$ into $\psi_{2}$ component that is decoupled from $\psi_{1}$ component in the absence of SOC. Since $\left[\mathcal{T}^{\prime}, \beta_{y} \sigma_{x} \hat{k}_{y}\right]=0$, the SOC component $\beta_{y} \sigma_{x} \hat{k}_{y}$ does not break newly defined time reversal symmetry $\mathcal{T}^{\prime}$ and cannot lead to topological effects.

The symmetry considerations for infinite lattice have direct implications for the formation of edge states in the truncated lattice illustrated in Fig. 2. Without SOC, $\beta_{x}=\beta_{y}=0$, one observes two bands touching in Dirac points at $k=K / 3$ and $k=2 K / 3$ that are remnants of the Dirac points of the bulk lattice. Two nontopological edge states arise at zigzag (red curve) and bearded (green curve) edges. When $\beta_{x}=0$ the SOC component $\sim \beta_{y}$ opens a gap that is nontopological in accordance with above considerations. In this gap one encounters only one nontopological edge state, whose energy is located in the gap (i.e. it does not connect two bands). Inclusion of weak SOC component $\sim \beta_{x}$ cannot immediately lead to appearance of topological edge states (second panel of Fig. 2): since nontopological gap already exists, it should first close under the action of $\beta_{x}$ component (this occurs around $\beta_{x}=0.85$, third panel of Fig. 2) and then reopen in the form of topological gap at $\beta_{x}>0.85$ (fourth panel, Fig. 2), where two topological modes connecting different bands appear.

A different scenario is observed when $\beta_{x}$ is large and one gradually increases $\beta_{y}$ contribution. At $\beta_{y}=0$ no gap exists in the spectrum even if $\beta_{x} \sim 1$, as was explained above. By adding even weak $\beta_{y} \sigma_{x} \hat{k}_{y}$ term into Hamiltonian, one breaks the time-reversal symmetry $\mathcal{T}$ and, hence, a topological gap appears (the Hamiltoian with $\beta_{x}>0$ does not obey $\mathcal{T}^{\prime}$ symmetry). The width of this gap monotonically increases with $\beta_{y}$, and unidirectional edge states exist at different edges, as shown in fifth and sixth panels of Fig. 2.

These results imply that both $\beta_{x}$ and $\beta_{y}$ SOC components are required for the existence of topological states. The largest topological gap was achieved for pure Rashba coupling, when $\beta_{x}=\beta_{y}$ (sixth panel of Fig. 2). Results presented in this figure constitute the central result of this paper: the emergence of topological edge states due to interplay of SOC and Zeeman lattice, induced by the inhomogeneous magnetic field.

Further we concentrate on the case of Rashba coupling $\beta_{x}=\beta_{y}$. Sixth panel of Fig. 2 indicates that edge states corresponding to zigzag (red branches) and bearded (green branches) edges may coexist for small intervals of Bloch momentum, but in general, they occupy different domains in $k$, that is similar to findings reported for single-component models [39]. Representative examples of topological edge states from zigzag and bearded edges are shown in Fig. 1. The best localization of the edge estate is achieved when energy $\varepsilon$ falls into the center of topological gap. When energy approaches lower or upper allowed bands, the mode strongly expands towards the center of the lattice.

Dispersive properties of the edge states are summarized in Fig. 3 where $k$-dependencies of derivatives $\varepsilon^{\prime} \equiv \partial \varepsilon / \partial k$ and $\varepsilon^{\prime \prime} \equiv \partial \varepsilon / \partial k$ are shown. Topological insulator usually supports unidirectional edge states, i.e. states at a given interface can move only in one direction, up or down. A peculiar property of our system is that edge states are not necessarily moving, i.e. the group velocity $\varepsilon^{\prime} \equiv \partial \varepsilon / \partial k$ can be zero for the zigzag edge modes and can even have two zero points for the bearded edge modes, as shown in Fig. 3(a). For quantum spin Hall states such zero group velocity modes on a conventional graphene lattices were observed in [40]. Thus, by changing Bloch momentum one can control the direction of the surface current. An unusual situation is possible when the Bloch number is in the vicinity of $k=K / 2$, where the states at the opposite edges move in the same direction (the red and green curves closely approach). Also, Fig. 3(b) suggests that second-order dispersion may vanish for some edge states. When broad envelope is superimposed on such a state, the latter moves along the interface over hundreds of lattice sites, representing linear quasi-non-dispersing wavepacket.

The example of topologically protected spinor surface current is shown in Fig. 4. We consider a triangular lattice with zigzag edges, with each edge containing 30 periods of the honeycomb structure. The initial state $\boldsymbol{\psi}(x, y)=\phi(x, y) e^{i k y} e^{-y^{2} / w^{2}}$ at $t=0$ was prepared using edge state $\phi$ corresponding to Bloch momentum $k=0.4 K$ with envelope of width $w=20$. Such a state can traverse several corners of triangular lattice returning to its original location. Although the triangular shape of the lattice is less favorable for persistent circular currents because of relatively strong scattering of edge states into the bulk modes at the corners, an almost complete round trip of the wave-packet still occurs.

Nonlinear edge states in SO-BEC. If atoms experience twobody interactions and one deals with a SO-BEC in a Zeeman lattice, the evolution of two-component (spinor) order parameter $\boldsymbol{\psi}$, is governed by the coupled Gross-Pitaevski equations:

$$
i \frac{\partial \boldsymbol{\psi}}{\partial t}=\frac{1}{2}\left(\frac{1}{i} \nabla+\mathbf{A}\right)^{2} \boldsymbol{\psi}+\hat{U}(\mathbf{r}) \boldsymbol{\psi}+g\left(\boldsymbol{\psi}^{\dagger} \boldsymbol{\psi}\right) \boldsymbol{\psi} .
$$

Here $\mathbf{A}=-\mathbf{i} \beta_{x} \sigma_{y}+\mathbf{j} \beta_{y} \sigma_{x}$ is the non-Abelian gauge potential, $g$ characterizes inter- and intra-species interactions, which are considered equal [24]. The sign of $g$ coincides with the sign of scattering length for two-body interactions and is considered relatively small, such that the BEC is in the superfluid phase for the chosen Zeeman lattice. Here we are interested in the impact of the nonlinearity on the proper- 

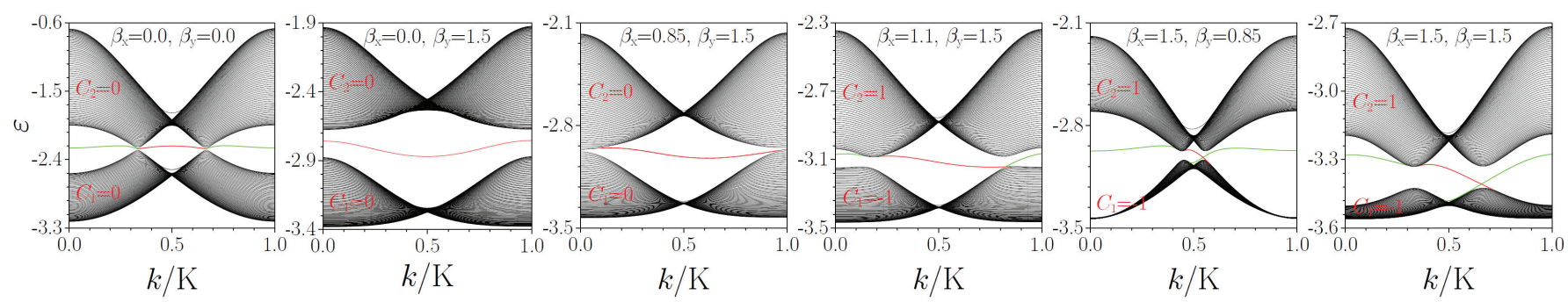

FIG. 2. Band-gap structures. Energy of linear states versus Bloch momentum $k$ in truncated Zeeman lattice with zigzag-bearded edges for different SOC strengths $\beta_{x}$ and $\beta_{y}$. Black curves correspond to bulk modes, while red (green) curves correspond to edge states residing on the left zigzag (right bearded) edge. The lattice parameters are $\rho=8, a=1.4$, and $d=0.5$. The Chern numbers $C_{1,2}$ of the two lowest bands of the respective bulk lattice are shown in the panels (see Methods).
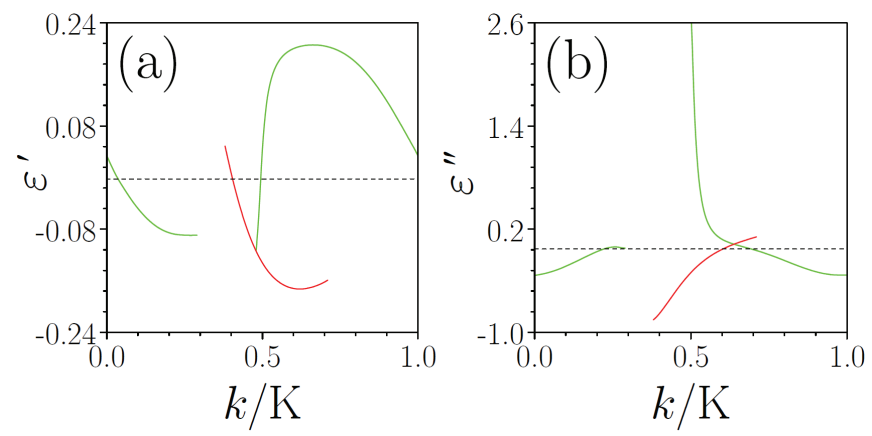

FIG. 3. Group velocity and Group velocity dispersion. Group velocity $\varepsilon^{\prime}$ (a) and dispersion $\varepsilon^{\prime \prime}$ (b) $v s$ Bloch momentum $k$ for edge states from zigzag (red) and bearded (green) edges at $\beta_{x}=\beta_{y}=$ 1.5. Dashed lines indicate $\varepsilon^{\prime}=0$ or $\varepsilon^{\prime \prime}=0$ level.

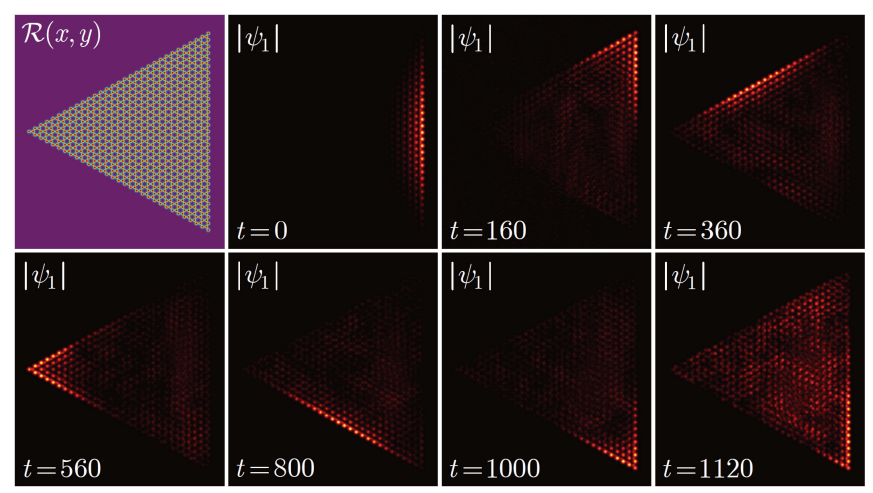

FIG. 4. example of persistent topologically protected spinor surface current. Honeycomb lattice with zigzag edges and $\left|\psi_{1}\right|$ distributions at different moments of time illustrating circulation of the edge state with broad envelope at $k=0.40 K, \beta_{x}=\beta_{y}=1.5$.

ties of topological edge states, considered above for the linear case. Topological states now are searched as nonlinear Bloch modes parameterized by the chemical potential $\mu$ and Bloch momentum $k: \psi(x, y)=e^{i k y-i \mu t} \phi(x, y)$. Bifurcations of such states from the linear edge states with $\mu=\varepsilon$ at the zigzag boundary are depicted in Fig. 5, where we plot the peak amplitudes $a_{1,2}$ of the spinor components and the number of atoms $N=\int_{-\infty}^{\infty} d x \int_{0}^{L} d y \boldsymbol{\psi}^{\dagger} \boldsymbol{\psi}$ per $y$-period, as functions of the chemical potential.
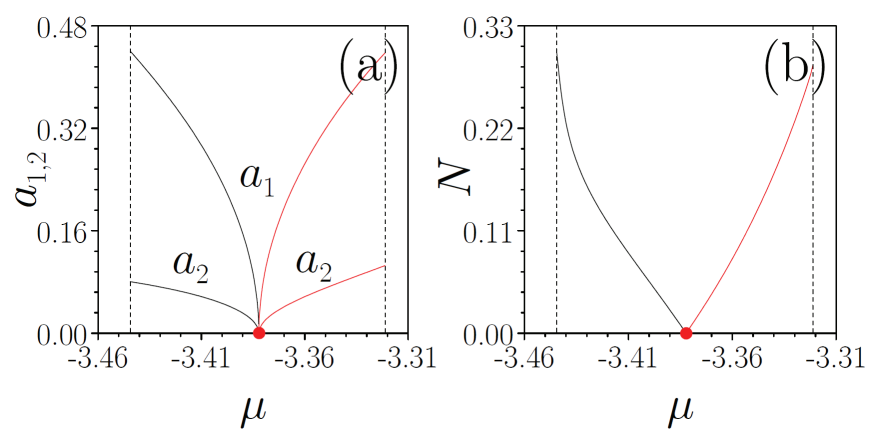

FIG. 5. Properties of the nonlinear edge states. Amplitudes of the components (a) and number of atoms per $y$-period (b) of nonlinear edge states at the zigzag edge versus chemical potential $\mu$ for $\beta_{x}=$ $\beta_{y}=1.5$. Black (red) curves correspond to $g=-1(g=+1)$. Vertical dashed lines indicate borders of the topological gap. Red dots correspond to linear edge states.

We considered positive, $g>0$, and negative, $g<0$, scattering lengths and found that amplitudes and norms of the nonlinear edge states monotonically increase toward the gap edges. They vanish in the point where they bifurcate from the linear edge state (red dots in Fig. 5). In all cases the first spinor component dominates. The ratio of the peak amplitudes $a_{1} / a_{2}$ slightly decreases towards upper edge of the topological gap. Both attractive and repulsive interactions lead to delocalization of the nonlinear edge states and their strong expansion into bulk of the lattice when the chemical potential approaches one of the topological gap edges.

Rigorously, nonlinear edge states in Zeeman lattices were found to be unstable. However, these instabilities may develop at very large evolution times, therefore in practice the states may be seen as metastable. For a topological edge state, instability is determined by the number of atoms $N$ and by the point of bifurcation of a nonlinear mode from the linear limit (the red dot in Fig. 5). Typical time scale of instability is larger for nonlinear modes bifurcating from the center of the gap and it decreases with $N$, when the chemical potential approaches a gap edge. For $g<0$ and $g>0$ instabilities are qualitatively different, as illustrated in Fig. 6 and Fig. 7, respectively. For selected value of $k=0.4 K$ the effective mass 


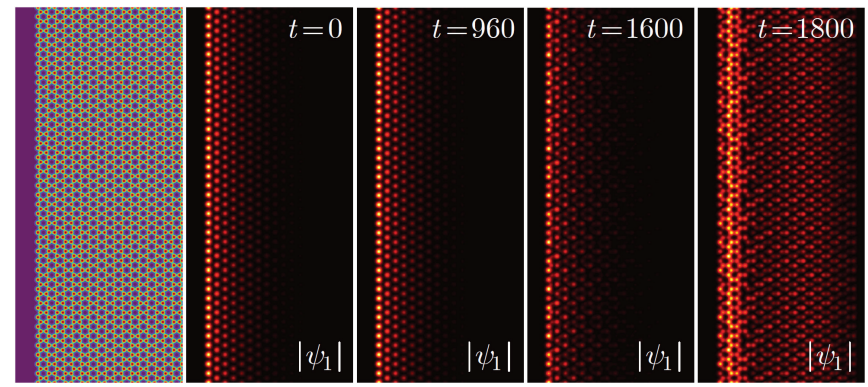

FIG. 6. Evolution of the nonlinear edge state. Evolution of the perturbed nonlinear edge state from zigzag edge at $\mu=-3.43, k=$ $0.40 K, \beta_{x}=\beta_{y}=1.5$, and $g=-1$.

$\sim 1 / \varepsilon^{\prime \prime}$ along the $y$-axis is negative. For $g<0$ this implies that no modulational instability can develop in the $y$-direction along the interface. Indeed, no splitting into regular fragments along the edge is observed in Fig. 6 and instability shows up as a dispersion of the edge state into the bulk. In the case of $g>0$ the development of modulational instability is possible for negative effective mass and one can clearly see fragmentation of the wave into periodic pattern in Fig. 7. This can be considered as a precursor to the formation of edge solitons.

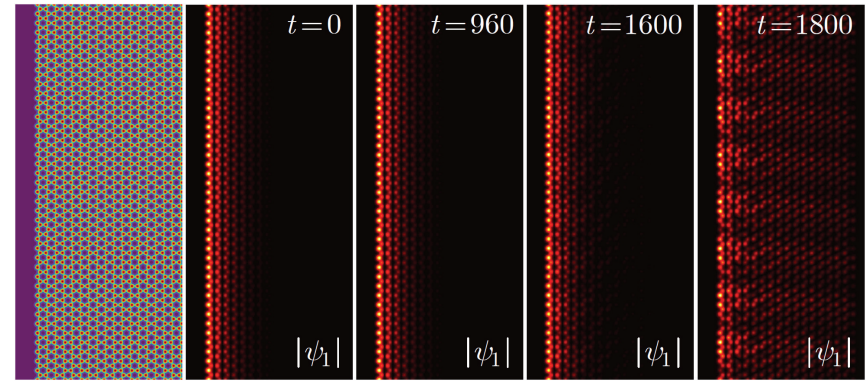

FIG. 7. Evolution of the nonlinear edge state. Evolution of the perturbed nonlinear edge state from zigzag edge at $\mu=-3.34, k=$ $0.40 K, \beta_{x}=\beta_{y}=1.5$, and $g=+1$.

Returning to the example of topologically protected spinor surface current illustrated in Fig. 4 for triangular structure, we verified that one round trip, similar to that depicted in Fig. 4, still occurs for moderate attractive and repulsive nonlinearities that only lead to increase of the bulk radiation.

\section{Discussion}

The reported results reveal the existence of topological edge states in atomic systems with SOC loaded in a honeycomb Zeeman lattice. We explored a wide range of parameters characterizing orthogonal components of the SOC and found that they play fundamentally different roles in the gap opening and edge state formation. The obtained states demonstrate persistent spinor surface currents in finite-size Zeeman lattices. When two-body interactions are included and the atomic system becomes a spin-orbit coupled BEC, nonlinear metastable topological edge states can exist.

While we report on the first ever example of topological edge states for atoms and BECs in Zeeman lattices, main ingredients required for observation of such states (spinorbit coupling with controllable Dresselhaus and Rashba contributions and Zeeman lattice) are already available and were realized in laboratories. Taking into account that atomic systems are widely recognized as excellent quantum simulators for diverse physical phenomena, we believe that the platform that we put forward opens an important new approach for realization of topological insulators in linear and nonlinear atomic systems. Therefore, our results allow for direct extension to other types of gauge fields which can be used to manipulate topological states.

\section{Methods}

The topological phase transition shown in Fig. 2 is confirmed by computing the Chern numbers of the respective bands [41, 42]:

$$
C_{n}=1 /(2 \pi) \int_{\mathrm{BZ}}\left[\partial_{x} A_{n y}(\mathbf{k})-\partial_{y} A_{n x}(\mathbf{k})\right] d^{2} k,
$$

where $A_{n \alpha}=i\left\langle\boldsymbol{\psi}_{n \mathbf{k}}\left|\partial_{k_{\alpha}}\right| \boldsymbol{\psi}_{n \mathbf{k}}\right\rangle$ is the Berry connection, $\boldsymbol{\psi}_{n \mathbf{k}}$ is the Bloch function of $n-$ th band, $\alpha=x, y$, and the integral is over the first Brillouin zone. Before the gap closing in Fig. 2, the lowest bands have equal Chern numbers $C_{1,2}=0$. After gap reopening the bands acquire Chern numbers $C_{1}=-1$ and $C_{2}=1$. According to bulk-edge correspondence this corresponds to a single topological edge state in the truncated lattice.

Data availability. The datasets generated during and/or analyzed during the current study are available from the corresponding author on reasonable request.
[1] Haldane, F. D. M. Model for a quantum Hall effect without Landau levels: Condensed-matter realization of the parity anomaly Phys. Rev. Lett. 61, 2015 (1988).

[2] Castro Neto, A. H., Guinea, F., Peres, N. M. R., Novoselov, K. S. \& Geim, A. K. The electronic properties of graphene. Rev. Mod. Phys. 81, 109 (2009).

[3] Kane, C. L. \& Mele, E. J. Topological order and the Quantum Spin Hall Effect in Graphene. Phys. Rev. Lett. 95, 226801 (2005).
[4] Hasan, M. Z. \& Kane, C. L. Colloquium: Topological insulators. Rev. Mod. Phys. 82, 3045 (2010).

[5] Qi, X.-L. \& Zhang, S.-C. Topological insulators and superconductors. Rev. Mod. Phys. 83, 1057 (2011).

[6] He, C. et al. Acoustic topological insulator and robust one-way sound transport. Nat. Phys. 12, 1124 (2016).

[7] Susstrunk, R. \& Huber, S. D. Observation of phononic helical edge states in a mechanical topological insulator. Science 349 (6243), 47 (2015). 
[8] Lu, L., Joannopoulos, J. D. \& Soljacic, M. Topological photonics. Nat. Photon. 8, 821 (2014).

[9] Haldane, F. D. M. \& Raghu, S. Possible realization of directional optical waveguides in photonic crystals with broken timereversal symmetry, Phys. Rev. Lett. 100, 013904 (2008).

[10] Raghu, S. \& Haldane, F. D. M. Analogs of quantum-Hall-effect edge states in photonic crystals, Phys. Rev. A 78, 33834 (2008).

[11] Wang, Z., Chong, Y., Joannopoulos, J. D. \& Soljačić, M. Observation of unidirectional backscattering-immune topological electromagnetic states. Nature 461, 772 (2009).

[12] Lindner, N. H., Refael, G. \& Galitski, V. Floquet topological insulator in semiconductor quantum wells. Nat. Phys. 7, 490 (2011).

[13] Hafezi, M., Demler, E. A., Lukin, M. D. \& Taylor, J. M. Robust optical delay lines with topological protection. Nat. Phys. 7, 907 (2011).

[14] Umucalilar, O. \& Carusotto, I. Fractional quantum Hall states of photons in an array of dissipative coupled cavities, Phys. Rev. Lett. 108, 206809 (2012).

[15] Khanikaev, A. B. et al. Photonic topological insulators. Nat. Mater. 12, 233 (2012).

[16] Rechtsman, M. C. et al. Photonic Floquet topological insulators. Nature 496, 196 (2013).

[17] Maczewsky, L. J., Zeuner, J. M., Nolte, S. \& Szameit, A. Observation of photonic anomalous Floquet topological insulators. Nat. Commun. 8, 13756 (2017).

[18] Mukherjee, S. et al. Experimental observation of anomalous topological edge modes in a slowly driven photonic lattice. Nat. Commun. 8, 13918 (2017).

[19] Bardyn, C.-E., Karzig, T., Refael, G. \& Liew, T. C. H. Chiral Bogoliubov excitations in nonlinear bosonic systems. Phys. Rev. B 93, 020502(R) (2016).

[20] Nalitov, V., Solnyshkov, D. D. \& Malpuech, G. Polariton Z topological insulator. Phys. Rev. Lett. 114, 116401 (2015).

[21] Karzig, T., Bardyn, C.-E., Lindner, N. H. \& Refael, G. Topological polaritons. Phys. Rev. X 5, 031001 (2015).

[22] Kartashov, Y. V. \& Skryabin, D. V. Modulational instability and solitary waves in polariton topological insulators. Optica 3, 1228 (2016).

[23] Kennedy, C. J. et al. Spin-Orbit Coupling and Quantum Spin Hall Effect for Neutral Atoms without Spin Flips, Phys. Rev. Lett. 111, 225301 (2013).

[24] Lin, Y.-J., Jiménez-García, K. \& Spielman, I. B. Spin-orbitcoupled Bose-Einstein condensates, Nature 471, 83 (2011).

[25] Galitski V. \& Spielman, I. B. Spin-orbit coupling in quantum gases. Nature 494, 49-54 (2013).

[26] Ruseckas, J., Juzeliunas, G., Öhberg, P. \& Fleischhauer, M. Non-Abelian Gauge Potentials for Ultracold Atoms with Degenerate Dark States. Phys. Rev. Lett. 95, 10404 (2005).

[27] Dalibard, J., Gerbier, F., Juzeliunas, G. \& Öhberg, P. Colloquium: Artificial gauge potentials for neutral atoms. Rev. Mod. Phys. 83, 1523 (2011).

[28] Beeler, M. C. et al. The spin Hall effect in a quantum gas, Nature 498, 201 (2013).

[29] Cooper, N. R. Optical flux lattices for ultracold atomic gases.
Phys. Rev. Lett. 106, 175301 (2011).

[30] Jiménez-García, K. et al. Peierls substitution in an engineered lattice potential. Phys. Rev. Lett. 108, 225303 (2012).

[31] Rashba, E. I. Properties of semiconductors with an extremum loop: 1. Cyclotron and combinational resonance in a magnetic field perpendicular to the plane of the loop. Fiz. Tverd. Tela (Leningrad),2, 1224 (1960) [Sov. Phys. Solid State 2,1109 (1960)].

[32] Dresselhaus, G., Phys. Rev. 100, 580 (1955)

[33] Galilo, B., Lee, D. K. K. \& Barnett, R., Topological Edge-State Manifestation of Interacting 2D Condensed Boson-Lattice Systems in a Harmonic Trap. Phys. Rev. Lett. 119, 203204 (2017).

[34] Radić, J., Di Ciolo, A., Sun, K. \& Galitski, V. Exotic Quantum Spin Models in Spin-Orbit-Coupled Mott Insulators, Phys. Rev. Lett. 109, 85303 (2012).

[35] Cole, W. S., Zhang, S., Paramekanti, A. \& Trivedi, N. BoseHubbard Models with Synthetic Spin-Orbit Coupling: Mott Insulators, Spin Textures, and Superfluidity, Phys. Rev. Lett. 109, 085302 (2012).

[36] Zarea M. \& Sandler, N. Rashba spin-orbit interaction in graphene and zigzag nanoribbons. Phys. Rev. B 79, 165442 (2009).

[37] Rakyta, P., Kormányos, A. \& Cserti, J. Trigonal warping and anisotropic band splitting in monolayer graphene due to Rashba spin-orbit coupling. Phys. Rev. B 82, 113405 (2010).

[38] Ablowitz M. J. \& Zhu, Yi. Evolution of Bloch-mode envelopes in two-dimensional generalized honeycomb lattices. Phys. Rev. A 82, 013840 (2010).

[39] Ablowitz, M. J., Curtis, C. W. \& Zhu, Yi. Localized nonlinear edge states in honeycomb lattices. Phys. Rev. A 88, 013850 (2013).

[40] Yang, Y. et al. Time-reversal-symmetry-broken quantum spin Hall effect, Phys. Rev. Lett. 107, 066602 (2011).

[41] Zak, J. Berry's phase for energy bands in solids. Phys. Rev. Lett. 62, 2747 (1989).

[42] Xiao, D., Chang, M.-C. \& Niu, Q. Berry phase effects on electronic properties. Rev. Mod. Phys. 82, 1959 (2010).

\section{ACKNOWLEDGMENTS}

Y.V.K. and L.T. acknowledge support from the Severo Ochoa Excellence Programme (SEV-2015-0522), Fundacio Privada Cellex, Fundacio Privada Mir-Puig, and CERCA/Generalitat de Catalunya. Y.V.K. acknowledges funding of this study by RFBR and DFG according to the research project No. 18-502-12080. V.V.K. was supported of the FCT (Portugal) under Grant No. UID/FIS/00618/2013. C. L. and F. Y acknowledge support from the NSFC (No. 61475101, No. 11690033 and No. 11805145).

Author contributions All authors contributed significantly to this work. 